\title{
Site educativo como tecnologia da informação e comunicação sobre o idoso com
}

\section{demência}

\author{
Educational site as information and communication technology on the elderly with dementia \\ Sitio educativo como tecnología de la información y la comunicación en ancianos con demência
}

Recebido: 28/06/2021 | Revisado: 06/07/2021 | Aceito: 08/07/2021 | Publicado: 18/07/2021

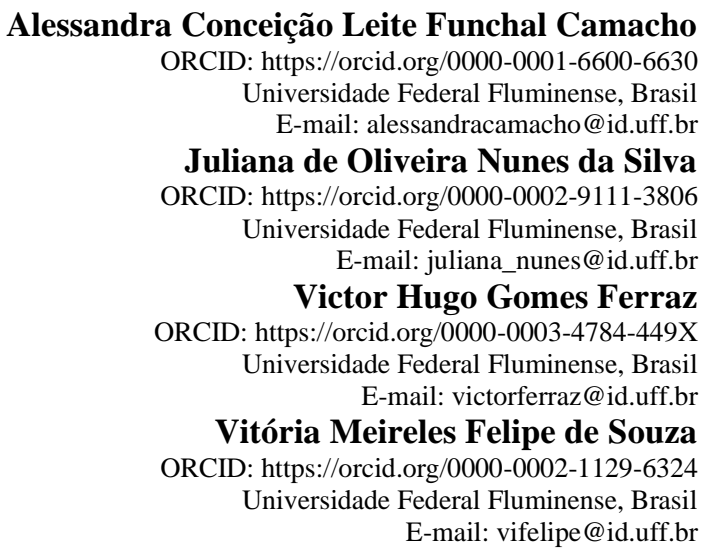

\section{Resumo}

Objetivo: descrever a interatividade do site educativo como tecnologia da informação e comunicação para o idoso com demência. Metodologia: trata-se de um estudo do tipo relato de experiência, com abordagem qualitativa do tipo descritiva desenvolvido através do projeto de extensão que acontece desde 2011 na Escola de Enfermagem Aurora de Afonso Costa (EEAAC) com o apoio e integração da Pró Reitoria de Extensão da Universidade Federal Fluminense. Resultados: este site como tecnologia educacional proporciona a divulgação e interatividade sobre orientações sobre a pessoa idosa com demência e seus cuidadores integrando o suporte a família e cuidadores de idosos com demências em período de Pandemia da Covid-19. Conclusão: este site educativo como tecnologia da informação e comunicação para a pessoa idosa com demência se trata de uma ferramenta que permite informar e estabelecer troca de conhecimentos, visando os cuidadores e familiares.

Palavras-chave: Saúde; Tecnologia da informação; Idoso.

\begin{abstract}
Objective: this paper aims to describe the educational website interactivity as information and communication technology for the elderly with dementia. Method: it is a study of the experience report type, with a qualitative descriptive approach, developed through the extension project that has been taking place since 2011 at the School of Nursing with the support and integration of the Dean of Extension of Federal Fluminense University. Results: this site as an educational technology provides dissemination and interactivity on guidance about elderly people with dementia and their caregivers, integrating support for families and caregivers of elderly people with dementia in times of the Covid-19 Pandemic. Final considerations: this educational site as information and communication technology for the elderly with dementia is a tool that allows for information and exchange of knowledge, aimed at family members and caregivers.
\end{abstract}

Keywords: Health; Information Technology; Elderly.

\section{Resumen}

Objetivo: este artículo tiene como objetivo describir la interactividad del sitio web educativo como tecnología de la información y la comunicación para personas mayores con demencia. Método: se trata de un estudio del tipo relato de experiencia, con enfoque cualitativo descriptivo, desarrollado a través del proyecto de extensión que se viene realizando desde 2011 en la Facultad de Enfermería con el apoyo e integración del Decano de Extensión de la Universidad Federal Fluminense. Resultados: este sitio como tecnología educativa brinda difusión e interactividad sobre orientación a personas mayores con demencia y sus cuidadores, integrando el apoyo a las familias y cuidadores de personas mayores con demencia en tiempos de la pandemia Covid-19. Consideraciones finales: este sitio educativo como tecnología de la información y la comunicación para personas mayores con demencia es una herramienta que permite la información y el intercambio de conocimientos, dirigida a familiares y cuidadores.

Palabras clave: Salud; Tecnología de la Información; Anciano. 


\section{Introdução}

De acordo com o Instituto Brasileiro de Geografia e Estatística (IBGE, 2020) cerca de 29 milhões de brasileiros apresenta 60 anos ou mais, cuja população corresponde atualmente a 13,5\% com perspectiva de crescimento para $24,5 \%$. Diante dessa realidade é verificado que as projeções de pessoas com demências também aumentem com uma proporção de mais de 45 milhões segundo dados disponibilizados pelo Instituto Alzheimer Brasil (IAB). Por serem acometimentos de ordem cerebral, as demências são doenças que causam diminuição do desempenho cognitivo, modificações importantes de comportamento e redução progressiva da capacidade funcional. Destaca-se a demência do tipo Alzheimer sendo a mais frequente na população idosa (SBGG, 2018).

Observa-se que com a transição demográfica e epidemiológica no Brasil, o processo de envelhecimento ocorreu de forma rápida, de maneira heterogênea nos diferentes municípios do país e no contexto de desigualdades sociais e culturais. Ademais, nesse contexto, predomina o modelo de atenção voltado para as demandas espontâneas, além de uma rede de apoio formal insuficiente e mudanças na estrutura familiar (Aires et al., 2020).

A partir dessa realidade, se verifica a insuficiência nos serviços de saúde para atender essa demanda crescente a exigir, no caso da Enfermagem, um cuidado qualificado a ser prestado a essa clientela. Com essa especificidade, tal situação nos coloca um verdadeiro desafio com o aumento do número de pessoas com demência devido à elevada expectativa de vida dos indivíduos.

Nesse contexto, aponta-se o cuidador familiar assumindo a função de cuidado repentina despreparado para o desempenho de seu novo papel. Não obstante, por uma demanda familiar ou por ausência de opções, essa pessoa é escolhida como cuidador necessitando de orientações relevantes para o desempenho dessa nova função (Nunes, Brito, Duarte, \& Lebrão, 2018).

Além disso, a família como fonte de suporte recorre para resolução de problemas não somente na rede de saúde mas também através de tecnologias educacionais onde procuram apoio a questões relevantes sobre o fato do cuidador ser idoso, escassa rede familiar, sobrecarga no cuidado, depressão, falta de disponibilidade para o cuidado em virtude de atividades laborais, impacto da doença, dificuldades no desenvolvimento do cuidado e apoio emocional (Camacho, Silva, \& Souza, 2020).

Destaca-se a boa funcionalidade familiar, por outro lado, que pode garantir aos idosos, mesmo dependentes, que continuem vivendo na comunidade, juntamente com seus familiares. Os profissionais de saúde devem orientar os cuidadores com as demandas de cuidados decorrentes da condição mental e funcional dos idosos para diminuir a sobrecarga a fim de reduzir o peso e o estresse e aumentar a qualidade de vida dos cuidadores e de seus familiares doentes. Os achados apontam a necessidade de políticas públicas que incluam as necessidades e o suporte aos cuidadores, em especial, os familiares de pessoas idosas, a fim de melhorar a qualidade de vida dos cuidadores familiares e a qualidade dos cuidados prestados às pessoas idosas (Nunes et al., 2018).

Assim, por meio das tecnologias de informação e comunicação se torna relevante desenvolver e divulgar pesquisas e informações importantes sobre a pessoa idosa com a Doença de Alzheimer e outros transtornos Demenciais e seus cuidadores, familiares com interatividade e de forma singular atentar para as demandas da realidade atual frente a Pandemia da Coronavirus Disease (Covid-19) instalada desde março de 2020. A divulgação permanente sobre as demências, auxilia na informação de cuidadores e familiares promovendo, através de uma tecnologia de informação e comunicação, a educação para a qualidade de vida do idoso demenciado. Bem como, favorece a participação ativa de cuidadores e de seus familiares, de forma a propiciar e incentivar o autocuidado, contribuindo para melhor sobrevida, bem-estar e o estado de equilíbrio de saúde da população idosa e seu cuidador (Camacho, Thimoteo, Souza, \& Silva, 2020). 
Destaca-se a interação entre aquele que divulga e quem acessa o conteúdo, possibilitando a troca de conhecimento de maneira a considerar a demanda do público alvo, isso a partir da aplicação de recursos digitais para divulgar conteúdos educacionais. Desse modo esta proposta de relato de experiência se enquadra no eixo 12 (Saúde do Idoso), subitem 12.5 (Análise da Gestão e das Práticas das Equipes no cuidado às especificidades em saúde da pessoa idosa) da Agenda nacional de prioridades de pesquisa em saúde (ANPPS) do Ministério da saúde, como relevância e justificativa por meio de tecnologia da comunicação e informação (Ministério da Saúde, 2018).

Nesse sentido, a proposta do site interativo como tecnologia educacional surgiu através da elaboração de um blog interativo que possui suas divulgações e atualizações constantes por meio das mídias sociais destacando a exposição de informações e conhecimentos acerca dos problemas presentes nos idosos diagnosticados com demências; a vulnerabilidade da pessoa com Transtornos de Demências como uma compreensão pelos cuidadores e/ou familiares, fato que é essencial a continuidade efetiva dos cuidados; bem como a rede de suporte aos cuidadores e/ou familiares. Como relevância e justificativa, dessa forma, procura-se por meio desta tecnologia, colaborar para aumentar o nível da qualidade de vida desses clientes, de forma a evidenciar a integralidade e humanização na assistência, bem como ações de prevenção, promoção, reabilitação e recuperação da saúde nos contextos comunitário, familiar e individual.

Diante dessas considerações, este artigo apresenta como objetivo descrever a interatividade do site educativo como tecnologia da informação e comunicação para o idoso que possui demência.

\section{Metodologia}

Trata-se de um estudo do tipo relato de experiência, com abordagem qualitativa do tipo descritiva desenvolvido por meio do projeto de extensão que ocorre desde 2011 na Escola de Enfermagem Aurora de Afonso Costa com o apoio e integração da Pró Reitoria de Extensão da Universidade Federal Fluminense. Esta Tecnologia da informação e comunicação foi estabelecida e ocorre atualmente promovendo conhecimentos acerca do idoso diagnosticado com ou sem demência, para seus familiares e cuidadores.

Caracteriza-se por possuir abordagem qualitativa e acadêmica, efetuada através de dados coletados diretamente no contexto de determinado acontecimento, através do uso de métodos de estudos de fenômeno social. Soma-se a isso, a descrição e análise detalhista de algum caso que apresente determinada particularidade, o tornando especial, assim, trata-se de um estudo de caso (Pereira, Shitsuka, Parreira, \& Shitsuka, 2018 apud Camacho, Souza, \& Menezes, 2021).

A partir disso, o presente artigo aborda essa ação de extensão que proporciona informações relevantes acerca dos cuidados de enfermagem efetuados a pessoa com Doença de Alzheimer assim como outros transtornos demenciais. Com a presença da Pandemia da Covid-19 instalada desde março de 2020, de 78.000 visualizações foi apresentada até a presente data 85.616 visualizações. Ou seja, ocorreu aumento superior a 7.616 visualizações de pessoas necessitando de informações relevantes sobre idosos e seus cuidadores sejam esses familiares ou cuidadores profissionais. São dúvidas e informações não somente sobre a temática, mas também relacionadas a Covid-19 nos idosos.

Em virtude dessa elevada demanda através do blog educativo (http://cicacamacho.blogspot.com/) com ampla divulgação nas rede sociais como Facebook e Instagram, através do projeto de extensão, optou-se também no ano de 2021 a criação do site sobre os cuidados de enfermagem desenvolvidos a pessoa com Doença de Alzheimer e outros transtornos demenciais (http://cuidadosalzheimer.uff.br) a fim de divulgar informações relevantes condizentes a demência na pessoa idosa articulando assuntos também relacionados a Covid-19.

Possui uma diretriz de interação dialógica que orienta o desenvolvimento de relações entre Universidade e setores sociais marcados pelo diálogo e troca de saberes sobre os tipos de demências no idoso. Portanto, ocorre participação de acadêmicos e docentes do curso de enfermagem da Universidade Federal Fluminense a fim de cooperar para a formação 
através da elaboração dos materiais de divulgação. Também se busca ampla divulgação nas redes sociais com o demonstrativo das figuras abaixo nos seus respectivos links de acesso no Facebook e Instagram:

Figura 1: Facebook - Cuidados à Pessoa com Doença de Alzheimer.
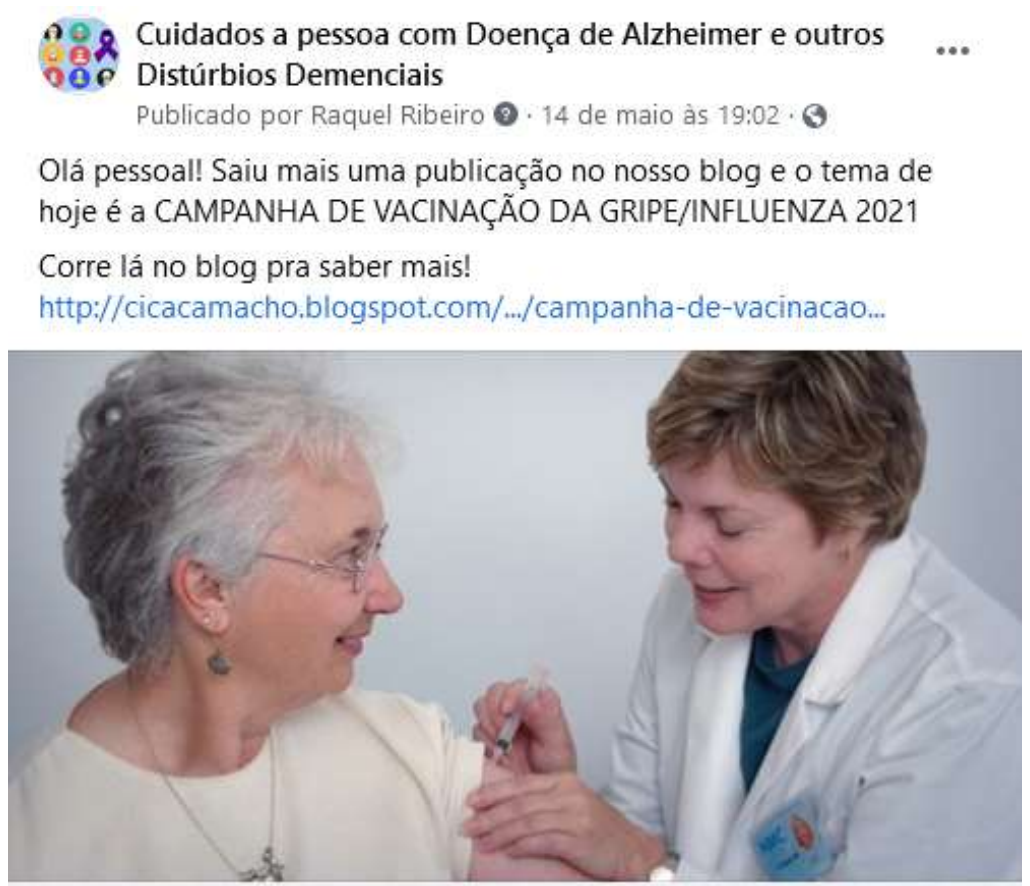

CICACAMACHO.BLOGSPOT.COM

CAMPANHA DE VACINAÇÃO GRIPE/NFLUENZA 2021

Fonte: https://www.facebook.com/cuidados.alzheimer/

Figura 2: Instagram - Cuidados à Pessoa com Doença de Alzheimer.

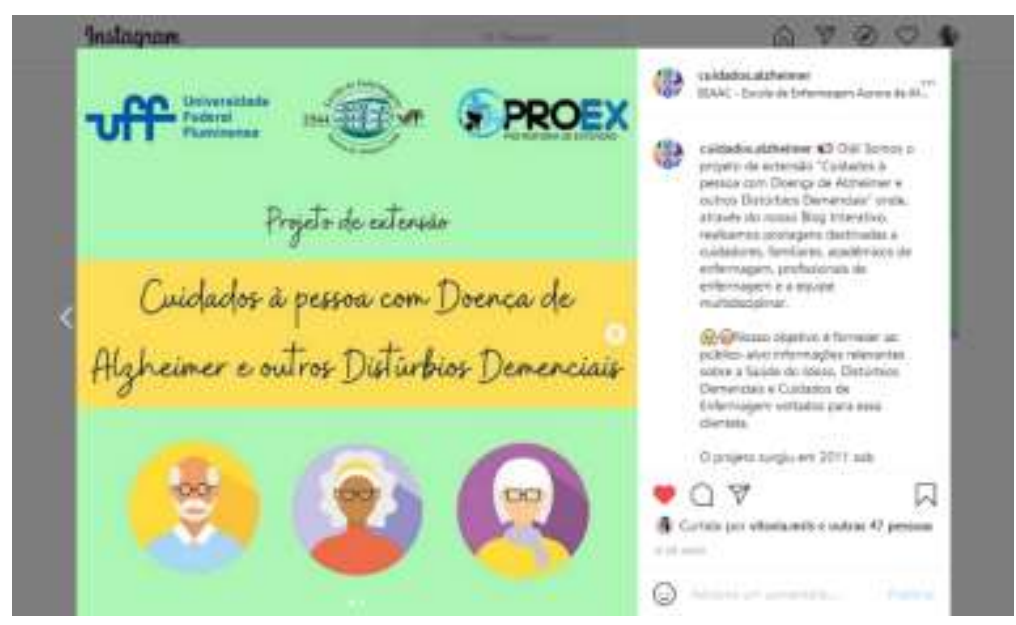

Fonte: https://www.instagram.com/cuidados.alzheimer/ (@cuidados.alzheimer)

A diretriz impacto e transformação social reafirma a Extensão Universitária como o mecanismo por meio do qual se estabelece a inter-relação da Universidade com os outros setores da sociedade, com uma atuação transformadora, voltada para os interesses e necessidades da maioria da população e propiciadora do desenvolvimento social e regional, assim como para o aprimoramento das políticas públicas. Nesse cenário, originou-se essa temática nas discussões e pesquisas realizadas no Grupo 
de Pesquisa Gestão da Formação e Qualificação Profissional: Educação e Saúde (GESPRO), que pertence ao Programa Acadêmico em Ciências do Cuidado em Saúde (PACCS) da Escola de Enfermagem Aurora de Afonso Costa (EEAAC), da Universidade Federal Fluminense (UFF).

\section{Resultados e Discussão}

\section{O envelhecimento e o Autocuidado do idoso}

Nota-se que conforme os aspectos teóricos de Wanda Horta (1971) da sua concepção até sua morte, o organismo humano passa pelo nascimento, puberdade, maturidade e chega ao envelhecimento. E é nessa fase natural e inevitável, que há o declínio das funções dos órgãos. Apesar de haver grandes controvérsias essa fase geralmente, inicia-se no final da segunda década da vida, perdurando por longo período e é pouco perceptível até surgirem no final dos 30 anos, as primeiras alterações fisiológicas e funcionais características do envelhecimento.

$\mathrm{Na}$ verdade, tanto os fatores biológicos quanto os sociais do envelhecimento citados acima, trazem consequências benéficas e também aquelas que podem trazer dificuldades para o ser que envelhece e que precisam ser analisadas pelos profissionais da saúde. Pode-se mencionar o déficit de autocuidado que ocorre conforme os indivíduos envelhecem e que pode ser exacerbado com a presença das doenças crônicas e degenerativas.

Acrescenta-se que é essencial considerar esta tecnologia interativa sobre as demências no idoso por meio de tecnologias de informática modernas como estratégia importante de acesso do público-alvo acerca dos cuidados desenvolvidos nas demências que incentivam a interatividade como processo contínuo de troca de conhecimentos e experiências (Camacho, Silva, \& Souza, 2020).

As condições de realizar as atividades de autocuidado são consideradas importantes para a população idosa, mesmo com a presença de doenças crônicas. Pode-se mencionar que o idoso se considera saudável quando realiza o autocuidado. Assim, observa-se uma relação de autocuidado, desenvolvidos pelos idosos, e a sua qualidade de vida. Esta relação deve ser alvo dos profissionais de saúde, entre eles o enfermeiro.

A maior parte dos cuidadores familiares utilizam a própria renda para cobrir os gastos com o idoso. Essa assistência toma formas de ajuda nas tarefas domésticas, nas atividades de vida diária, nas despesas com medicamentos e alimentação. Neste contexto de limitação da geração de renda, somado ao elevado custo para manter o cuidado ao idoso dependente no domicílio, acredita-se que os cuidadores tenham o convívio social e lazer prejudicados, o que influencia para acentuar a sobrecarga de cuidado (Aires et al., 2020)

No entanto, para obtenção de informações relevantes sobre o autocuidado do idoso através de seus cuidadores Santos, Silveira e Camacho (2016) destacam que a incorporação das tecnologias da informação e comunicação desencadeia modificações nas relações com o outro, o mundo e o conhecimento, interferindo na subjetividade do indivíduo, em que o homem passa a usufruir das novas tecnologias, compartilhando-as e as utilizando em sua vida cotidiana, algumas, como no caso o computador, o celular com acesso às redes sociais e à internet, que adentram o lar com papel relevante na vida dos indivíduos que as utilizam.

Haja vista que o ambiente familiar se constitui como uma principal fonte de apoio ao idoso, há que se estimular o fortalecimento das relações familiares com o propósito de se minimizarem as dificuldades e angústias vivenciadas por ambos, familiares e idosos (Camacho \& Coelho, 2010).

Percebe-se a fundamental importância da compreensão do conteúdo sobre a qualidade de vida na velhice, visto que, é central ao desenvolvimento de iniciativas de prevenção e intervenção, objetivando à promoção e à reabilitação nos vários contextos da vida do indivíduo, além de também auxiliar na avaliação e no planejamento de serviços e políticas destinados a promover o bem-estar de idosos. 


\section{Tecnologia da informação e comunicação acerca do idoso diagnosticado com demência}

Esta tecnologia interativa sobre as demências no idoso por meio de tecnologias de informática modernas nos levou a considerá-la como uma estratégia importante de acesso dos cidadãos acerca dos cuidados desenvolvidos nas demências que incentivam a interatividade como processo contínuo de troca de informações.

Nesse contexto, foram debatidos os assuntos vinculados aos cuidados fundamentais de Enfermagem, sobretudo cuidados específicos focados nas doenças crônicas, como nutrição oral, quedas/biossegurança, higiene corporal, conforto; hábitos de vida, eliminações fecal e urinária, suporte nas medicações; déficit de comportamento e o suporte ao cuidador/ família. Isso porque se busca orientar e auxiliar os cuidados de enfermagem ao indivíduo diagnosticado com a doença de Alzheimer e outros transtornos demenciais (Queiroz \& Camacho, 2016). São destaques:

\section{- Resgate e valorização das reminiscências e da identidade pessoal do idoso:}

Aponta-se ênfase sobre a diminuição da capacidade da memória na demência. Mas a memória não é apenas uma função cognitiva, ela é forjada de conteúdos pessoais e sociais. A reminiscência, como estratégia de intervenção, consiste, assim num processo psicológico de recuperação de experiências pessoais, vividas no passado que são utilizadas para fins terapêuticos (Lopes, Afonso, \& Ribeiro, 2014).

Portanto, carrega a identidade de uma pessoa, de um grupo, de uma geração. O idoso demenciado, mesmo com dificuldades na memória pode ainda se lembrar de diversos aspectos da sua vida e o resgate dessas reminiscências é um importante objetivo a ser atingido. Algumas oficinas, como o grupo de conversas e a oficina da memória se voltam para esse fim. Pode-se utilizar diversos recursos para estimular as reminiscências, como fotografias, músicas, figuras, relatos de familiares, entre outros. Ao colocarmos a pessoa em contato com a sua própria história de vida, reforçamos o seu sentimento de identidade pessoal e sua autoestima.

\section{- Estimulação cognitiva e das habilidades sensório-motoras}

A estimulação cognitiva é uma importante ferramenta para a reabilitação (seja relativa ou temporária) das habilidades cognitivas das pessoas com demência. A atenção, o raciocínio lógico, a memória e a linguagem são algumas das funções que se visa estimular. Campo fundamental para a elaboração de atividades por fonoaudiólogos, terapeutas ocupacionais, psicólogos e outros.

Essa busca por um envelhecimento com maior qualidade se mostra evidente a elevada procura de atividades que possibilitam o bem-estar e que, consequentemente, estejam relacionadas ao cuidado da saúde física e mental. A motivação para esta busca de atividades saudáveis pode estar relacionada ao receio do idoso de adquirir doenças que afetam a sua independência e autonomia (Abrahão \&, Camacho, 2020).

Diversos são os materiais, estratégias e atividades que se pode utilizar para a estimulação cognitiva: jogos, figuras, formas, leitura, escrita, desenhos, pinturas, confecção de cartazes, objetos manufaturados, música, sons, cores, odores, materiais táteis e outros. Dentre as oficinas que exploram esse viés, destacam-se a oficina da palavra, a oficina de jogos e notícias, a oficina de expressão e os grupos de estimulação cognitiva.

\section{- Atividades com os cuidadores}

$\mathrm{O}$ acolhimento e a orientação ao familiar e/ou cuidador são tão importantes quanto o atendimento ao paciente referido. "Cuidar de quem cuida" é trabalhar em prol da qualidade de vida dos pacientes e seus familiares. Essa é uma importante ferramenta para auxiliá-los a lidar com as tensões e dificuldades da função que exercem. Como lidar com o paciente na hora do banho, agitação, agressividade e esclarecimentos sobre a doença são alguns dos temas que serão abordados. Os familiares 
desconhecem a razão das dificuldades dos pacientes gerando situações de negligência ou até mesmo violência, por parte de familiares e/ou seus cuidadores. Além do suporte dos profissionais, o próprio grupo funcionará como um espaço de troca de experiências e apoio mútuo.

Haja visto, geralmente as tecnologias de comunicação e informação são um crescente recurso que possibilita suporte virtual àquele indivíduo que precisa de determinada compreensão sobre os cuidados aos idosos diagnosticados com demência.

Abaixo destaca-se por meio do link de divulgação uma figura representativa de uma das postagens realizadas sobre os cuidados ao idoso com demência:

Figura 3: Postagem de divulgação de informações acerca dos cuidados ao idoso diagnosticado com demência.

\section{TIPOS DE DEMÊNCIA}
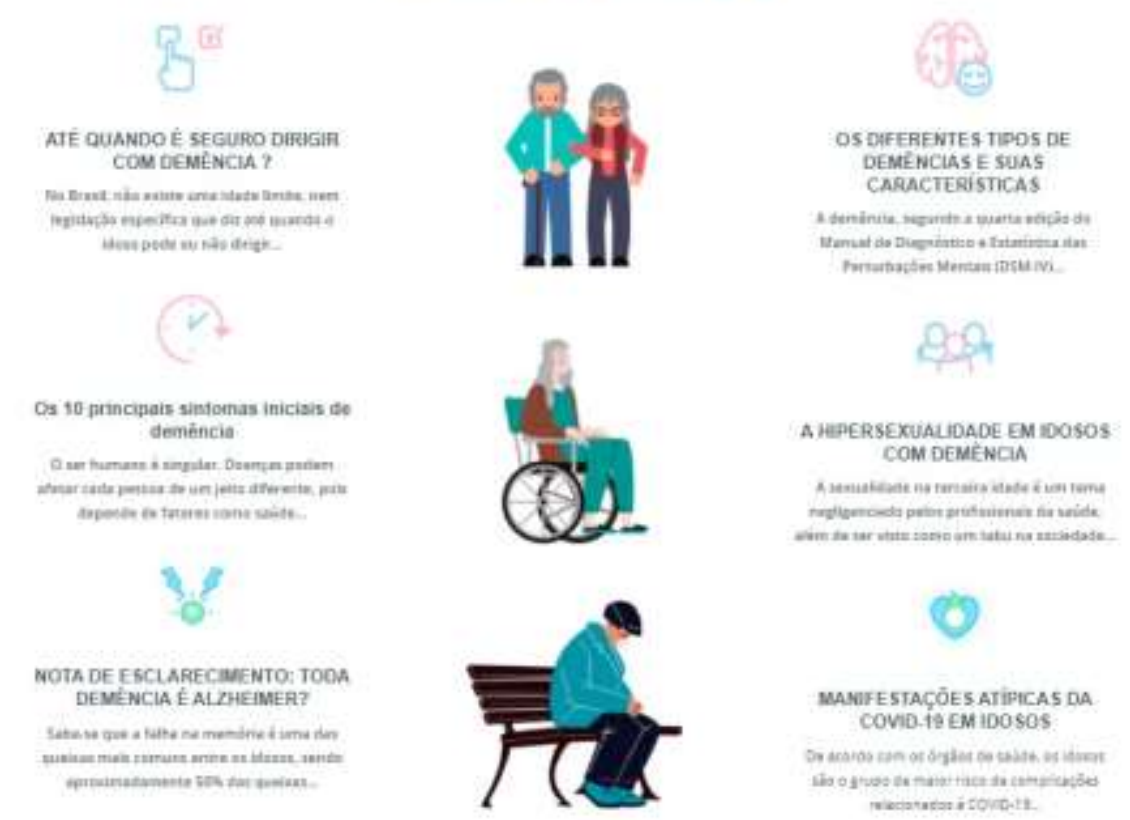

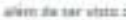
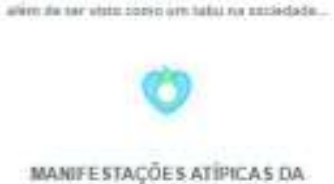

COVID. fe EM iDOSOS

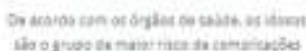

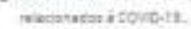

Fonte: Site Cuidados a pessoa com Doença de Alzheimer e outros transtornos demenciais: http://cuidadosalzheimer.uff.br

Os cuidadores precisam de mais informações sobre o manejo da doença e como lidar com os fatores estressantes devido à evolução da doença. As tecnologias podem ser úteis na promoção da interação sugerindo acompanhar os pacientes e apoiar os familiares dentro do caminho de cuidados, além de coletar e compartilhar informações entre os diferentes profissionais de saúde envolvidos. Novos estudos devem ser direcionados para investigar se o uso de estratégias educacionais podem ser a abordagem certa para atender às necessidades das famílias que convivem com demência (Webster et al 2017).

As atividades desenvolvidas nesta tecnologia possibilitam oportunidades de engajamento institucional que aceitem e estimulem a atuação dos familiares/cuidadores de maneira a possibilitar a buscar a independência e autonomia do idoso demenciado. Assim como, realizar a educação para impactar positivamente na qualidade de vida, e proporciona a participação ativa do cuidador e familiares do idoso com demência, ocasionando e estimulando o autocuidado, a contribuir para melhor sobrevida.

Nota-se a atuação essencial do suporte informacional aos familiares/cuidadores visto que se torna essencial para a situação de saúde e equilíbrio dos idosos com demência, assim como o estadiamento ocasionado pelo transtorno e os cuidados que são necessários por causa das limitações efetuadas pela doença. 
Nesse cenário, tal experiência abordada nesta ação de extensão é capaz de ser ampliada a fim de atingir diversos contextos, e assim empregar em outros assuntos de interesses da saúde para serem usados em comunidades nas práticas educativas relacionadas à saúde, uma vez que, trata-se de uma estratégia tecnológica que usa linguagem simples, de maneira atrativa e interativa.

Santos et al (2020) apontam a necessidade de estimular a implementação de modificações nas estratégias e maneira de abordar os usuários, bem como o uso de novas tecnologias de comunicação e informação, a fim de tornar as ações eficazes para a modificação do estilo de vida e melhoria das condições de saúde e qualidade de vida dos pacientes.

Tal tecnologia se caracteriza pela acessibilidade de maneira a atender os direitos preconizados pelas políticas de saúde, tanto para a pessoa idosa, cuidador e familiar, assim como, dúvidas sobre alimentação e cuidados de higiene relacionados aos idosos com demências. Acrescenta-se a importância da incorporação de tecnologias, aplicando novos métodos de educação em saúde que colabora para o cuidado aos idosos com demências.

Neste sentido, as atividades desenvolvidas por meio das tecnologias educacionais podem atender a uma educação em saúde baseada em ações que reconhecem as verdadeiras necessidades, desejos e aspirações de familiares/cuidadores, bem como no ensino acadêmico da enfermagem.

\section{A tecnologia da Informação e Comunicação em tempos de pandemia da COVID-19}

Aponta-se a pandemia da Covid-19 que restringiu e ocasionou novas configurações sociais, sobretudo relacionado aos idosos. A vista disso, os familiares de indivíduos com intensa aproximação apresentam papel fundamental nas atitudes que visam apoio a fim de diminuir os intensos impactos na vida dessa população (Camacho, Silva, \& Souza, 2020). As informações fornecidas aos cuidadores/familiares possuem grande relevância, pois remetem as questões do cotidiano vinculadas a situação de saúde do idoso.

Segundo a Organização Mundial de Saúde (OMS), a pandemia da Covid-19 ocasiona cerca de 15\% de letalidade e o conjunto de idosos com comorbidades possui risco elevado de complicações (OMS, 2020).

Não obstante os idosos com Doença de Alzheimer com a Covid-19, precisam de maior atenção nos cuidados, porque podem manifestar comprometimento do quadro funcional e cognitivo (ABRAz, 2020). Outrossim, os sinais e sintomas da Covid-19 são expostos pelo Ministério da saúde (2020) como sensação de cansaço para atividades de rotina, falta de ar, tosse, augesia (perda do paladar) e, a anosmia (perda do olfato) em alguns pacientes.

Diante dessa realidade à procura de informações relacionadas aos cuidados efetuados na população idosa demenciada durante a Pandemia da Covid-19 por meio das mídias sociais aumentaram e assim, despertou interesse por conhecimentos nas mídias digitais sobre essa enfermidade.

Há inúmeros relatos de cuidadores sobre o grau de disfunção cognitiva após contrair a doença. Para indivíduos com 50 anos ou mais, as sequelas da Covid-19 tem sido mais intensas e ocasionado comprometimentos neurológicos e prejudicado habilidades mentais. Estes comprometimentos podem ser um fator de risco para o desenvolvimento de demências, como a Doença de Alzheimer (Brasil, 2021).

Abaixo destaca-se por meio do link de divulgação uma figura representativa de uma das postagens realizadas nesta época de Pandemia pelo COVID-19: 
Figura 4: Postagem de divulgação de informações sobre Pandemia pela Covid-19.

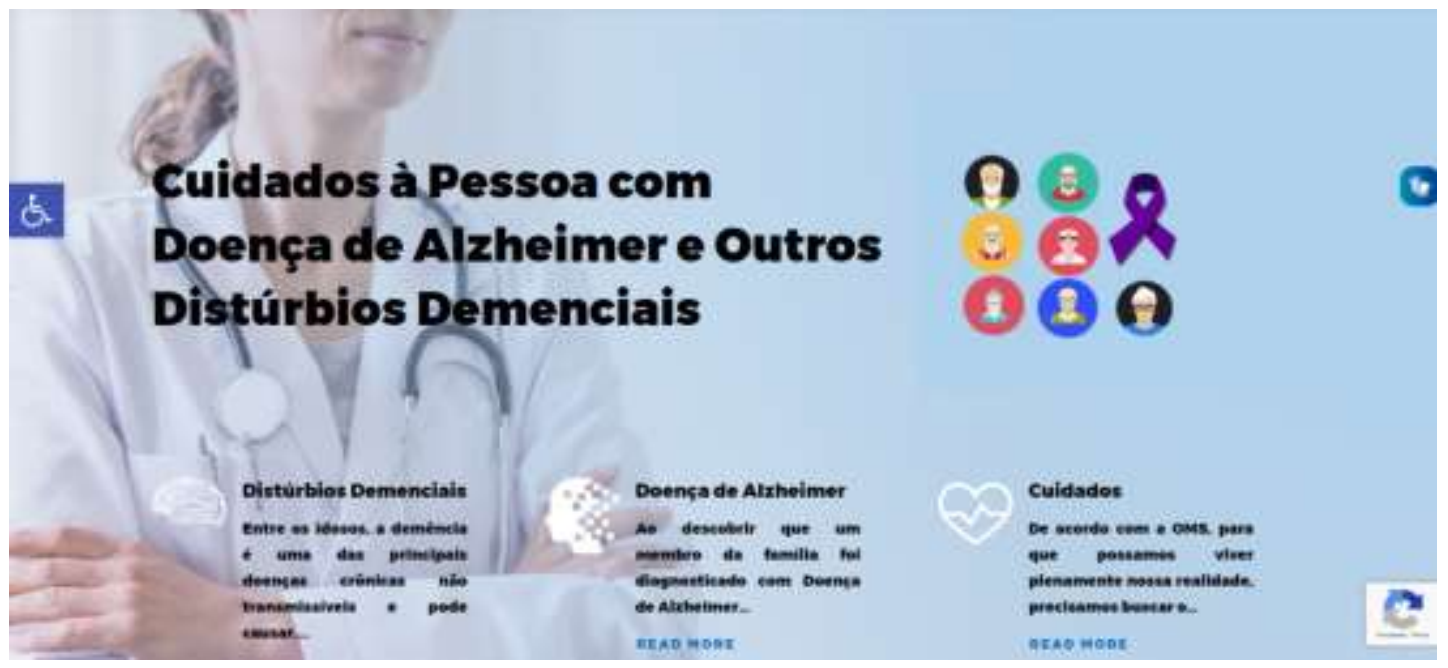

Fonte: Site Cuidados a pessoa com Doença de Alzheimer e outros transtornos demenciais: http://cuidadosalzheimer.uff.br

Segundo a Sociedade Brasileira de Geriatria e Gerontologia (SBGG) a vacinação também é destaque quando falado da importância de vacinar idosos para reduzir a mortalidade nessa faixa etária, principalmente as pessoas idosas com comorbidades e idosos com 80 anos ou mais que possuem a síndrome de fragilidade, sendo então essencial a realização das ações de distanciamento e isolamento social (SBGG, 2021).

Nesse contexto, a elaboração dos ambientes de aprendizagem interdisciplinares e cooperativos, apresentam como objetivo o fornecimento das informações acerca da população idosa durante a pandemia. A vista disso, as tecnologias educacionais em saúde são um recurso essencial, já que estão inseridas no cotidiano da pessoa idosa e dos cuidadores formais ou familiares, assim, possibilita a construção do conhecimento de maneira didática, dinâmica e interativa. Além disso, importante ressaltar a potencialização das informações adquiridas pelos visitantes dessa tecnologia educacional, bem como à interação e os debates, sendo um instrumento de educação em saúde (Camacho, Silva, \& Souza, 2020).

Observa-se ainda a permissão da independência e autonomia no processo interativo, de forma a incentivar a participação dos cuidadores/ familiares com acesso simples e prático, além de permitir ações vinculadas a promoção da educação em saúde, refletindo um dos diversos papéis da Enfermagem junto a essa população em tempos de isolamento e distanciamento social pela pandemia (Camacho, Silva, \& Souza, 2020).

Nesse cenário, se torna imprescindível estimular modificações nas estratégias e na maneira de abordagem dos usuários, a partir do uso de novas tecnologias educacionais. Tal fato, ocasiona maior eficácia nas ações educativas na área da saúde a fim de incentivar mudança do estilo de vida e melhoria das condições de saúde e por consequência, da qualidade de vida (Santos et al., 2020).

O site como tecnologia educacional na área da saúde permite a compreensão dos direitos vinculados as políticas públicas de saúde, alimentação, cuidados de higiene, e precauções importantes acerca da Covid-19 vinculadas aos idosos e seus familiares, sobretudo, questões relacionadas ao isolamento social, importância da higienização das mãos, informações sobre atividades lúdicas para a pessoa idosa, assim como a importância da vacinação contra a Covid-19 e da influenza.

\section{Considerações Finais}

Com o objetivo de descrever a interatividade do site educativo como tecnologia da comunicação e informação para a pessoa idosa diagnosticada com demência é oportuno o destaque que essa tecnologia educacional possibilita uma compreensão 
sobre as pessoas idosas com demências vivenciados no cotidiano no sentido de possibilitar a melhoria do estado de equilíbrio e a qualidade de vida, sobretudo durante a pandemia da Covid-19 que determina a necessidade de distanciamento e isolamento social, tornando assim acessível as informações imprescindíveis para a saúde do idoso. Esse relato de experiência trouxe o desenvolvimento e a importância dessa Tecnologia Educacional, na qual se tornou possível oferecer conteúdos relacionados à saúde desse idoso demenciado aos seus cuidadores.

A limitação desse trabalho está vinculada na inacessibilidade a essas tecnologias por outros usuários em período de isolamento social, visto que não são todos que apresentam acesso à internet, redes sociais ou até mesmo computadores e smarthphones. Além disso, como recomendação, orienta-se a utilização dessa tecnologia como maneira de informar e dar sentido à racionalidade educativa, sobretudo, nas regiões do país em que o período de isolamento social ainda persistem

Como sugestão, enfatiza-se a importância de adotar tecnologias educativas em saúde, incorporadas no ensino acadêmico. E, neste caso, essa tecnologia oferece uma gama de relevantes recursos para a educação em saúde voltadas para esse público.

\section{Referências}

Abrahão, L. A., \& Camacho, A. C. L. F. (2020). A importância do enfermeiro no estímulo cognitivo e de memória em pessoas idosas: revisão integrativa. Revista Research, Society and Development, 9(7), 1-19, e306974180, 2020.

ABRAz. Associação Brasileira de Alzheimer. (2020). COVID-19: O novo coronavírus e a doença de Alzheimer. ABRAZ, http://abraz.org.br/web/2020 /03/13/covid-19-o-no vo-coronavirus-e-a-doenca-de-alzheimer/

ABRAz. Associação Brasileira de Alzheimer. (2021). Estimulação cognitiva para a prevenção de sequelas da COVID-19.

ABRAz, https://abraz.org.br/2020/2021/02/05/estimulacao-cognitiva-para-a-prevencao-de-sequelas-da-covid-19/.

Aires, M., et al. Sobrecarga de cuidadores informais de idosos dependentes na comunidade em municípios de pequeno porte. Rev Gaúcha Enferm, 41(esp), e20190156.

Brasil. Ministério da Saúde. (2018). Agenda nacional de prioridades de pesquisa em saúde (ANPPS). Brasília: Ministério da Saúde. http://bvsms.saude.gov.br/bvs/publicacoes/agenda_prioridades_pesquisa_ms.pdf

Brasil. Ministério da Saúde. (2020). Secretária de Vigilância em Saúde: Boletim epidemiológico. Brasília: Ministério da Saúde. http://portalarquivos2.saude.gov.br/images/pdf/2020/janeiro/23/Boletim_epidemiologico_SVS_04.pdf

Camacho, A. C. L. F, \& Coelho, M. J. (2010). Políticas públicas para a saúde do idoso: revisão sistemática políticas públicas para a saúde do idoso: revisão sistemática. Rev Bras Enferm., 63(2), 279-284.

Camacho, A. C. L. F., Silva, R. T., \& Souza, V. M. F. (2020). Tecnologia da informação ao idoso em tempos de COVID-19. Research, Society and Development, 9(6), e124963497.

Camacho, A. C. L. F., Thimoteo, R. S., Souza, V. M. F., Silva, R. P., \& Silva, M. A. P. (2020). A importância da interatividade para idosos com demências através das tecnologias da informação. Research, Society and Development, 9(4), e134942975.

Horta, W. A. (1979). Processo de Enfermagem. EPU.

Horta, W. A., Hara, Y., \& Paula, N. S. (1971). O Ensino dos Instrumentos Básicos de Enfermagem. Rev. Bras. Enf., 24(3), 159-169.

IBGE. Instituto Brasileiro de Geografia e Estatística. (2020). Projeção da população do Brasil e das Unidades da Federação. IBGE, https://www.ibge.gov.br/apps/populacao/projecao/

Lopes, T., Afonso, R., \& Ribeiro, O. (2014). Impacto de intervenções de reminiscência em idosos com demência: revisão da literatura. Psic., Saúde \& Doenças, 15(3), 597-611.

Nunes, D. P., Brito, T. R. P., Duarte, Y. A. O., \& Lebrão, M. L. (2018). Cuidadores de idosos e tensão excessiva associada ao cuidado: evidências do estudo sabe. Rev Bras Epidemiol, 21(suppl 2), E180020.SUPL.2.

OMS. Organização Mundial da Saúde (2020). Coronavirus disease 2019 (COVID-19): Situation Report -51. OMS, https://www.who.int/docs/defaultsource/coronaviruse/situation-reports/20200311-sitrep-51-covid-19.pdf?sfvrsn=1ba62e57_10

Queiroz, R. S., \& Camacho, A. C. L. F. (2016). Educational technology on care to elderly with dementia: experience report. Revista enfermagem UFPE online, 10(Suppl.1), 361-365.

Santos, A. S., Silveira, R. E., \& Camacho, A. C. L. F. (2016). A Inserção Digital na Terceira Idade. In Campos, A. C. V., Berlezi, E. M. \& Correa, A. H. M. (Orgs.), Teorias e Práticas Socioculturais na Promoção do Envelhecimento Ativo (Vol.5). (pp.219-242). Editora Unijuí, 2016. 
Research, Society and Development, v. 10, n. 8, e58410817651, 2021

(CC BY 4.0) | ISSN 2525-3409 | DOI: http://dx.doi.org/10.33448/rsd-v10i8.17651

Santos, L. H. R., et al. (2020). Grupo educativo e qualidade de vida de idosos hipertensos e diabéticos. Research, Society and Development, 9(1), e178911893.

SBGG. Sociedade Brasileira de Geriatria e Gerontologia. (2019). Em Dia Mundial do Alzheimer, dados ainda são subestimados, apesar de avanços no diagnóstico e tratamento da doença. SBGG, https://sbgg.org.br/em-dia-mundial-do-alzheimer-dados-ainda-sao-subestimados-apesar-de-avancos-nodiagnostico-e-tratamento-da-doenca/

SBGG. Sociedade Brasileira de Geriatria e Gerontologia. (2021). Congresso Brasileiro de Geriatria debate a importância das vacinas. SBGG, https://sbgg.org.br/congresso-brasileiro-de-geriatria-debate-a-importancia-das-vacinas/

Webster, L., et al. (2017). Development of a core outcome set for disease modification trials in mild to moderate dementia: a systematic review, patient and public consultation and consensus recommendations. Health Technol Assess, 21(26), 1-192. 